\title{
When do arrows start to compete? A developmental mouse-tracking study
}

\author{
Frouke Hermens \\ University of Lincoln, UK
}

\begin{abstract}
Recent work in adults has suggested that the strength of social and symbolic cues not presented at fixation (but allowing eye movements to the cue) may be determined less by their biological relevance and more by the distinctiveness of the shape of the cue. The present study examines whether these results extend to children, who may differ in their relative exposure to symbolic cues (arrows) compared to social cues. Children aged 3 to 11 were presented with congruent or incongruent pairs of cues (line drawings of gazing eyes, pointing hands, and arrows) and were asked to indicate the direction of the target cue (indicated at the start of the block) by moving the mouse towards the response box indicating its direction. Results show a similar advantage for arrows and pointing hands in young children as previously found in adults, suggesting that cue shape trumps biological relevance for cues away from fixation from an early age.
\end{abstract}

Keywords: Social attention, mouse tracking, symbolic cueing 


\section{Introduction}

The development of responses to social cues (such as eye gaze and pointing gestures) has been studied extensively (for an overview, see Frischen, Bayliss, \& Tipper, 2007). For example, it has been shown that 3 months old children follow head gaze shifts (D'Entremont, Hains, \& Muir, 1997) and eye gaze cues (Hood, Willen, \& Driver, 1998). Joint attention, where children show evidence of understanding another person's attention, is thought to develop from around 12 months of age (Butterworth \& Jarrett, 1991). Such gaze cueing effects are a marker of the understanding of others' beliefs and thoughts, known as theory of mind, which has been suggested to develop later at around four years of age (Baron-Cohen, 1995). The influence of cues provided by others has received much attention, because it may provide a distinction between learned (symbolic cues) and hard-wired (social cues) cueing effects (Baron-Cohen, 1995), and reduced responses to social cues are considered to be an important aspect of autism spectrum disorders (Hoehl et al., 2009).

Studies of the effects of social cues, however, have often been restricted to cues presented where the observer is already looking (e.g,. by replacing a fixation dot by the face stimulus or by presenting the face stimulus in the middle of the screen, Driver et al., 1999; Kuhn \& Kingstone, 2009). In day-to-day viewing, such immediate fixations of social cue do not always occur, and instead observers first look somewhere else, after which they make an eye movement to the social cue. In a recent study, we have shown that in such a situation, adults show a reduced effect of gaze cues compared to arrow cues or pointing hands (Hermens, Bindemann, \& Burton, 2017). In the experiments, participants were presented with two cues, placed above and below the fixation point (similar to Figure 1b). Before the start of the block, participants received an instruction indicating which cue they had to respond to (e.g., 'In this block, always respond to the direction of the arrow cue'), but

Dr Frouke hermens, School of Psychology, University of Lincoln, Brayford Pool, Lincoln, Lincolnshire, LN6 7TS, United Kingdom, E-mail: fhermens@lincoln.ac.uk. The author wishes to thank Holly Allaway for invaluable help in running the study, Fenja Ziegler and Zoe Maed for organizing the Summer Scientist event, and Ferenc Igali, Heather Shaw and Chris Luke for technical support. 
participants did not know where this target cue was going to appear (above or below the fixation point). Eye tracking during the task suggested that participants therefore adopted a strategy in which they first looked at the fixation point until the cues appeared, and then made an eye movement to the target. In these experiments, faster response times were found to arrow cues, pointing hands and rotated heads, and slower response times to gaze cues embedded within a face, gazing eyes in isolation, and words indicating a direction (e.g., 'LEFT'). The experiments also examined whether the other cues in each pair interfered with responses (when the non-target cue was pointing in the opposite direction). Such interference effects were weak when response times were considered. However, when participants responded by moving a mouse cursor to a target box (i.e., they engaged in a mouse tracking paradigm, Freeman \& Ambady, 2010), mouse trajectories deviated strongly in the direction of incongruent arrow, pointing hand, and rotated head cues, while no such effects on mouse trajectories were found from gazing eyes (embedded in a face, or in isolation) or direction words. Together these findings suggest that cues with a clearly visible directional shape that can be seen away from fixation (e.g., arrows, pointing hands) exert the strongest influences on participants' responses. These shape related effects appear to be independent of the social or biological relevance of the cues, and occur even when participants can make an eye movement to the cue (Hermens et al., 2017). Distraction effects of simultaneously presented cues occur, but may only be revealed by mouse trajectories.

The results by Hermens et al. (2017) agree with the findings of other studies that have looked at the effects of social and symbolic cues presented away from fixation. For example, Burton, Bindemann, Langton, Schweinberger, and Jenkins (2009) used a paradigm in which one cue was presented at fixation (the cue that had to be responded to) and another cue away from fixation. They found that rotated heads and pointing hands interfered with responses to gazing eyes, but that gazing eyes did not interfere with responses to pointing hands and rotated heads. In their study, the cues were presented for a short amount of time, so that participants could not make an eye movement, but the experiments by Hermens et al. (2017) with the same cues suggest that Burton et al. (2009) would have found the same 
results if they had left the stimuli on the screen and allowed participants to make an eye movement to the distractor stimulus. Similar results were found by Langton (2000), who found for competing cues provided by a single actor (e.g., looking up while pointing down) head-gaze cues and arrows both influenced responses to pointing gestures. Interestingly, Nummenmaa and Hietanen (2009) found similarly strong cueing effects from gazing eyes and arrows, but they also reported that the strength of cueing appeared to be linked to the exact stimulus used.

All these studies have considered adults, who have strong exposure to arrows in day to day life and may therefore be primed to respond to these cues by associating them with a direction. In children, exposure to arrow cues may be reduced compared to adults. While there does not seem to be documented evidence that children encounter and look at arrows less often than gaze shifts and pointing gestures, there are indicators that arrows may be weaker cues in children than in adults, and that gaze cues provide strong cueing in this population. For example, Senju, Tojo, Dairoku, and Hasegawa (2004) found that while children (average age $=11.1$ years) were sensitive to unpredictive distractor arrows (with a $50 \%$ cue validity), no evidence of cueing was found when the arrows were counterpredictive (20\% cue validity). Gaze cues did influence children's responses under both conditions. In adults, counterpredicitve cues have been found to yield equally strong cueing as gaze cues (Tipples, 2008). Likewise, Jakobsen, Frick, and Simpson (2013) found that infants (9 months old) and young children (3-4 years old) were driven by the perceptual weight distribution of the central cue (with square endings having similar cueing effects as arrowheads), while older children (5-6 years) old and adults (19 years old) were driven by the shape of the cues. This suggests that for younger children, cues that are unbalanced should yield strong cueing, independent of the shape of the cue. Not all studies, however, have found stronger responses to gaze cues than to arrows in children. For example, Kuhn et al. (2010) found similar cueing effects from arrows in adults and older children (10 years old) in an oculomotor task, and Barnes, Kaplan, and Vaidya (2007) found interference from arrows in both younger (6-9 years old) and older children (10-13 years) when responding to centrally presented 
direction words (LEFT/RIGHT). Likewise, Ristic, Friesen, and Kingstone (2002) found cueing from arrows in young children (3-5 years old), when responding to the appearance of a peripherally presented target. All these studies have found that gaze cues are at least as strong as arrows, but also have in common that the cues were presented at fixation.

The present study investigates whether the results in adults (Hermens et al., 2017), where arrow cues and pointing hands had stronger influences than gazing eyes when not presented at fixation, extend to children, who can be assumed to have weaker exposure to arrows. Children (3 to 11 years) performed a mouse tracking task (Freeman \& Ambady, 2010), in which they responded to a target cue, presented simultaneously with a distractor cue (Figure 1). Mouse tracking was used, because it was shown previously that mouse trajectories reveal influences of simultaneously presented distractor cues that may not be detected with response times (Hermens et al., 2017). Three cues were compared: eye gaze, pointing hands (both biologically relevant) and arrows (a symbolic cue). If the results in children mimic the results in adults (Hermens et al., 2017), stronger influences (as targets on response times, as distractors on mouse trajectories) are expected for hand and arrow cues. If exposure to a cue is the main driver of the strength of a cue, it can be expected that younger children (aged 3-5 years) show stronger cueing to social (eye gaze and pointing hands) than to symbolic cues (arrows). With increasing age, exposure to arrows can be expected to increase, and the difference in cueing effects between social and symbolic cues is expected to decrease in older age groups if exposure is driving the cueing effects. The exact shape of the function linking age to cueing effects for the different types of cues is difficult to predict, because the exact exposure to the different types of cues is unknown, and it is unclear how much exposure is needed to achieve optimal cueing, and whether stronger exposure automatically lead to stronger attention (cues that are encountered less may draw more attention, simply because of novelty of the stimulus). 
Methods

\section{Participants}

Participants were children taking part in the Summer Scientist event organized by the psychology department of the University of Lincoln (UK). Children aged between 3 and 11 years old took part in return for tokens that they could spend on games, such as Super Mario Kart and face painting. 150 children took part in the study (81 female, 69 male, mean age $=6.3$ years, $\mathrm{sd}=1.93)$. The experimental protocol was approved by the local ethics committee.

\section{Apparatus}

A dual core Dell computer with Windows 7 and a 19 inch Dell flat screen were used for data collection, in combination with a standard optical USB mouse. The computer was placed on a table so that enough space was available to move the mouse around without hitting the keyboard or the edge of the table. The mouse speed was set to one level below the standard mouse speed (middle setting) of Windows 7. The software was programmed in OpenSesame (Mathôt, Schreij, \& Theeuwes, 2012), using a modified version of the script provided by Eoin Travers.

\section{Stimuli}

Three images were used as targets, which could either point or look to the left or right. These were a hand, an arrow, or a pair of gazing eyes (Figure 1a), all 3.4 degrees of visual angle wide. In the top corners of the screen, two blue buttons (measuring 4.4 by 2.4 degrees) with slightly smaller versions of these target images were shown (Figure 1b). In the bottom of the screen, 4.5 degrees away from the screen's bottom edge, a light blue start button (measuring 4.4 by 1.3 degrees) was shown, created using Microsoft Powerpoint, with the word 'START!' in capital letters printed on it. Upon clicking the button, it disappeared and was immediately replaced by the target and distractor, placed 2 degrees above and below the position of the button. A standard black Windows mouse cursor (a small left upward 
pointing arrow shape, 0.3 degrees in length) was used as the cursor for the experiment.

\section{Design}

Participants performed 5 practice trials followed by 48 experimental trials. The first five practice trials were using the same target as the 16 trials in the next block, but varied the distractor congruency and identity. In each block of 16 trials, one of the three targets (hand, arrow, or eyes) was used, and one of the two remaining cues was used as a distractor. For example, when the target was the arrow in the block, 8 trials would use a hand and 8 trials would use the eyes as the distractor stimulus. The target was equally often presented above or below the start button, and was equally often pointing to the left or to the right. The distractor was equally often in the same ('congruent') or in the opposite ('incongruent') direction of the target. The order of the trials within each block was randomized for each participant, and the order of the targets was counterbalanced across participants in the order of entering the study (using one of the six possible orders).

\section{Procedure}

Children were seated at a desk on a small chair positioned at a distance of around $75 \mathrm{~cm}$ from the screen, and held the mouse in their preferred hand. The experimenter and sometimes one of the parents were sitting on each side of the table to provide instructions and moral support to keep going with the study. Before the start of the experiment, the software asked for the participant's gender (using Mickey and Minnie Mouse images) and age (in years). The first target was then shown on the screen, together with instructions. If children could read, they read these instructions themselves, but often, the experimenter skipped the instruction screen, and instead demonstrated one or two of the practice trials while explaining the task. Children were explained that their task was to decide whether the target was pointing or looking to the left or right, and to indicate this by clicking one of two response buttons with the mouse positioned in the two top corners of the screen. They could reveal the target and distractor item by clicking a 'START!' button positioned in the center and slightly above the bottom of the screen. Clicking this button made the 
a) Stimuli
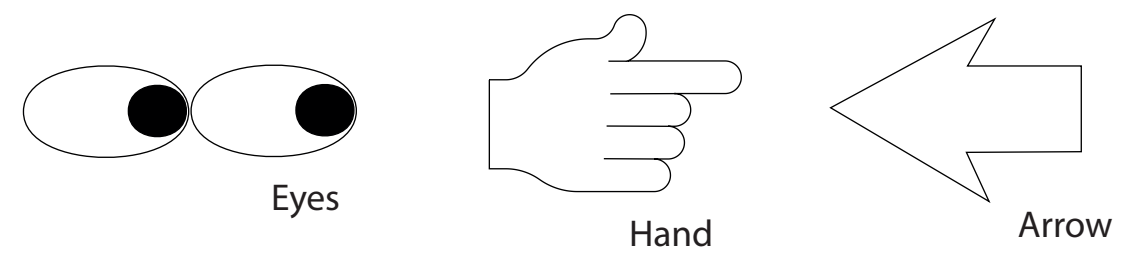

b) Stimulus sequence

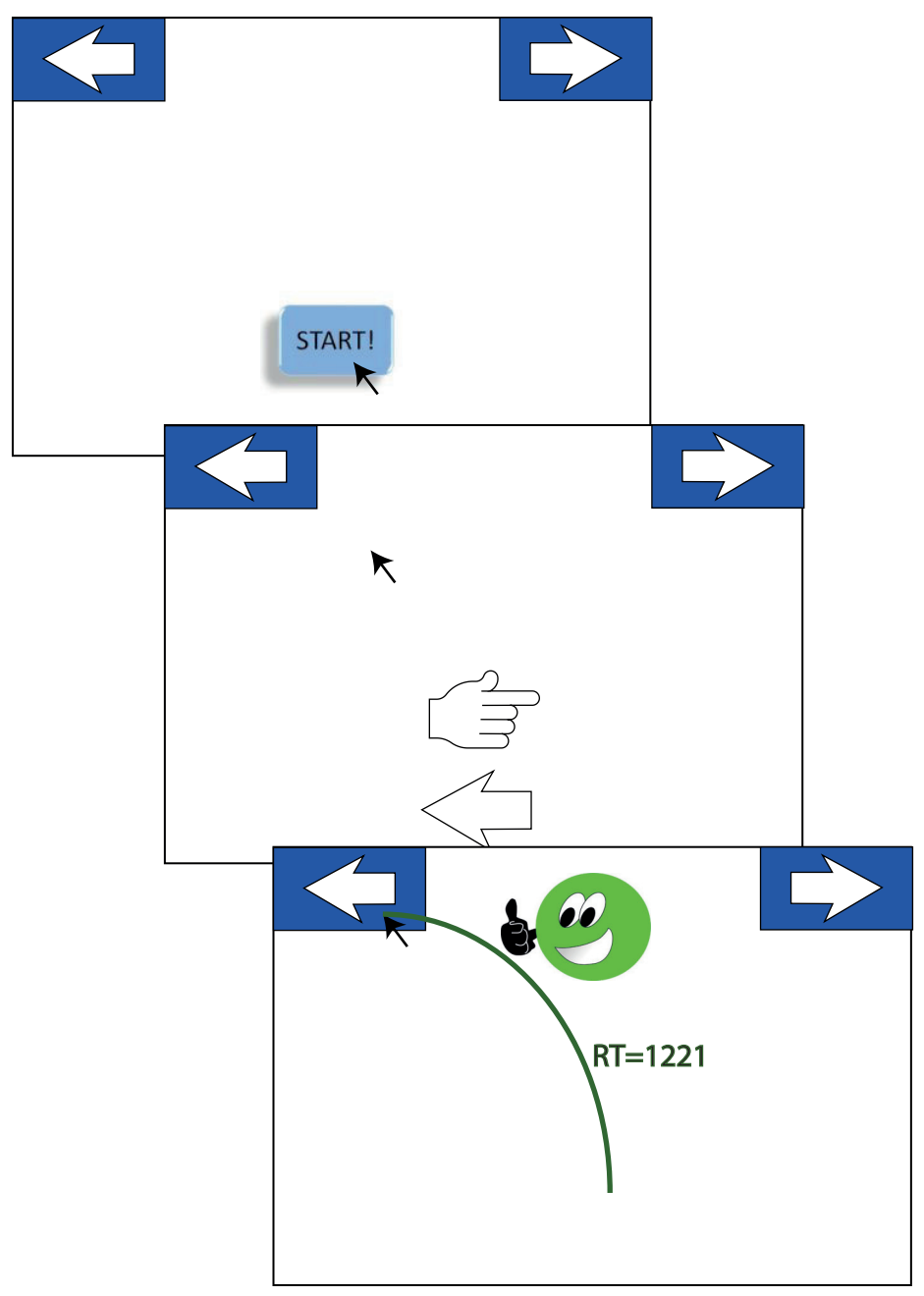

Figure 1. a) The stimuli in the experiment. On each trial, the target (one of the three stimuli, defined at the start of the block) was shown together with a distractor (one of the remaining stimuli). b) Stimulus sequence. Participants started with a screen with a start button in the bottom of the screen and two response buttons in the top of the screen showing the target in that block. After clicking with the mouse on the start button, two stimuli were presented above and below where the start button was shown (which was removed from the screen immediately after the mouse click). One of these stimuli was the target shown in the two response boxes at the top of the screen (an arrow in this case). Participants were instructed to indicate the direction of the target by moving the mouse cursor to the corresponding box in the top of the screen and clicking the mouse. A feedback screen showed the mouse trajectory, the response time, and a smiley face. Green colors were used when the response was correct, whereas feedback was shown in red for an incorrect response. For incorrect responses, the smiley face looked unhappy and was pointing its thumb downwards. 
button go away and the stimuli appear immediately. The two target buttons were visible throughout the experiment. Before each block of trials, a screen introduced the new target for that block, showing an image of the target and a text indicating a new block was about to start.

\section{Data analysis}

Six measures were extracted from the mouse trajectories. The three response time measures are illustrated in Figure 2a. Initiation times were defined at the time needed to leave the start button. Movement times were defined as the time between leaving the start button and entering the clicked box. Finally, completion times were defined as the time between clicking the start button and entering the response box. For all three measures, median response time per child were used in the statistical analysis and the plotting of the data, to avoid extremely long response times influencing the average data (without the need of deciding what outlier procedures to use). Response time measures were based on all correct experimental trials (practice trials excluded). In standard versions of mouse tracking (Freeman \& Ambady, 2010), participants are encouraged to start moving the mouse as quickly as possible by an error message if they fail to do so. For children, we did not use such an error message, because response times were found to vary hugely across the age groups, which would have meant that younger children (with the slowest response times) would constantly receive error messages. Removing the error message for slow starts does mean that some of the response time effects could enter the initiation times, and possibly reduce mouse trajectory effects, which is why several response time measures were analyzed.

Three further measures were derived from the mouse trajectories: (1) the maximum deviation between the mouse trajectory and the straight line connecting the start button click and the response box click (as a percentage of the length of this straight line), (2) the time to peak (the moment at which the maximum deviation occurred, as a percentage of the length the straight line connecting the start button click and the response box click), and (3) the percentage of turn-around trajectories. Maximum deviation was used as the main 
measure of attraction towards the alternative responses, because this distance can be easily expressed as a percentage of the straight line length. As a consequence, maximum deviation is independent of the size of the screen without the need to rescale the screen to a standard size before computing the measures (as is the case for the alternative measure, area under the curve, AUC, Freeman \& Ambady, 2010). For saccade trajactories, maximum deviation and AUC have been shown to be highly correlated (Ludwig \& Gilchrist, 2002), and similar results have been reported for mouse trajectories (Freeman \& Ambady, 2010). Negative values for maximum deviations indicate deviations away from the alternative response, whereas positive values indicate deviations towards the alternative (attraction). Time to peak can be defined in different ways. Here we use the spatial position along the straight line where maximum deviation occurs, providing a measure of where in the trajectory the maximum deviation occurs independent of speeding up or slowing down of the mouse movement. To complement maximum deviations, a second measure of attraction to the alternative response box was used, focusing on the trajectories that deviated strongly towards the alternative. Inspection of the histogram of maximum deviations suggests that the majority of deviations was smaller than $40 \%$, but a substantial number of trajectories had larger deviations. The percentage of turn-around trajectories quantifies, for each stimulus condition, the percentage of trials with such large deviations. This measure is different from the 'x-flips' and ' $y$-flips' measures sometimes used (Dale, Roche, Snyder, \& McCall, 2008), that are thought to be a measure of mouse trajectory complexity (Freeman \& Ambady, 2010). Because some children had difficulties clicking, but not moving the mouse, the trajectory measures were based on the trajectory from leaving the START button and the moment of entering the final response box (the part of the trajectory measured by the movement times).

Because the study did not employ a full 3 (targets) by 3 (distractors) by 2 (congruency) design (when presenting two of the same cues on a single trial, it would be unclear which cue to respond to), and because the effects of target and distractor may be confounded when conducting a 3 (targets) by 2 (distractors) by 2 (congruency) ANOVA on the data, separate analyses are performed to test statistically for the observed patterns in the 
data. The first set of statistical analyses focused on the strengths of the cues (eyes, hand or arrow) independent of age. In a first analysis, the strength of each type of cue as a target ('how easy is it to respond to eyes, hands, and targets, independent of the distracting cue?') was assessed by pooling median response times for each participant across distractors and congruency conditions, after which the resulting numbers were compared using Bonferroni corrected t-tests (ANOVAs were skipped, because significant differences were expected on the basis of earlier results Hermens et al., 2017). In a second analysis, the relative strengths effects of each of the two cues for each target (e.g., 'are arrows stronger distractors than hands when responding to eyes?') were compared using the interaction effect in a two-way repeated measures ANOVAs testing the effects of distractor type and distractor congruency. Finally, Bonferroni corrected follow-up t-tests comparing congruent and incongruent conditions for each target-distractor combination examined the distraction effect for each distractor separately (e.g., 'do incongruent hands significantly interfere with eyes?'). To avoid long lists of results, these latter effects will be shown as p-values in the graphs.

In a second set of statistical analyses, the influence of age on the distractor effects were assessed. The first, more general analysis starts with each target-distractor combination (six combinations in total) as one factor, and congruency and age as the other two factors. This analysis will demonstrate whether general age effects can be found. The second, more specific analysis examines whether congruency interacts with age for each target-distractor combination separately. These latter results will be shown as p-values in the data plots.

Each time when multiple comparisons are performed (e.g., t-tests for each targetdistractor combination), a Bonferroni correction for the number of comparisons will be applied. For ease of interpretation of the results, the p-values will be multiplied by the number of comparisons, so that values of less than $p=0.05$ will denote significant effects after Bonferroni correction. In some instances, multiplication of the p-values led to values above 1. Because p-values do not reach values above one, the notation $p>0.99$ will be used in those instances. 
Results

From the 150 children who took part in the study, 134 completed the study (see Figure $2 \mathrm{~b}$ ). Common reasons for withdrawing from the task were being bored with the task and struggling to use the mouse. Children withdrawing early from the study were often younger $\left(\chi^{2}=65.0, p<0.0001\right.$; comparing children younger than 6 years and 6 years or older), but there were no differences completion rates between males and females $\left(\chi^{2}=\right.$ 0.52, $p=0.47)$. Subsequent analyses were performed for the children who completed all trials $(\mathrm{N}=134)$. To ensure sufficiently large group sizes, data from children aged 3 and 4 were combined into a new category ("4-"), as well as the data from children aged 10 and 11 ("10+"). The resulting group sizes were 16 (for 3 and 4 years), 25 (5 years), 25 (6 years), 28 (7 years), 19 (8 years). 13 (9 years) and 8 (10 and 11 years).

Figure 2b shows that accuracy $(F(8,125)=7.75, p<0.001)$ and response times $(F(8,125)=31.0, p<0.001)$ both depended on age, showing an exponential-like decrement in response times with age and an inverse exponential approach towards perfect performance. The longer response times may explain why younger participants did not always complete the experiment.

Response time measures

Initiation times. Data plots for the first response time measure, initiation time (how long participants took to move the mouse out of the START box), are shown in Figure 3. It took children more time to move the mouse out of the START box for eye-gaze stimuli than for hands (independent of the distractor, $t(133)=2.64, p=0.027, d=0.23$ ) and arrows $(t(133)=2.40, p=0.053, d=0.21)$. The time to move out of the START box did not differ for hands and arrows $(t(133)=0.030, p>0.99, d=0.0026)$.

Distraction effects of arrows and hands on eyes did not differ significantly (no interaction between cue type and congruency, $\left.F(1,133)=1.47, p=0.68, \eta^{2}=0.011\right)$, and neither cue had significant distraction effects on initiation times ( $p$-values in Figure 3a). Distraction effects of hands were stronger than those of eyes when responding to arrows (interaction: 


\section{a) Data analysis}

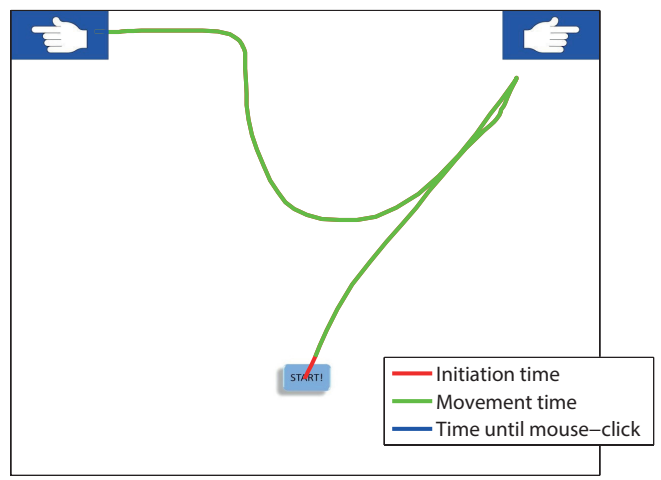

\section{b) Demographics}
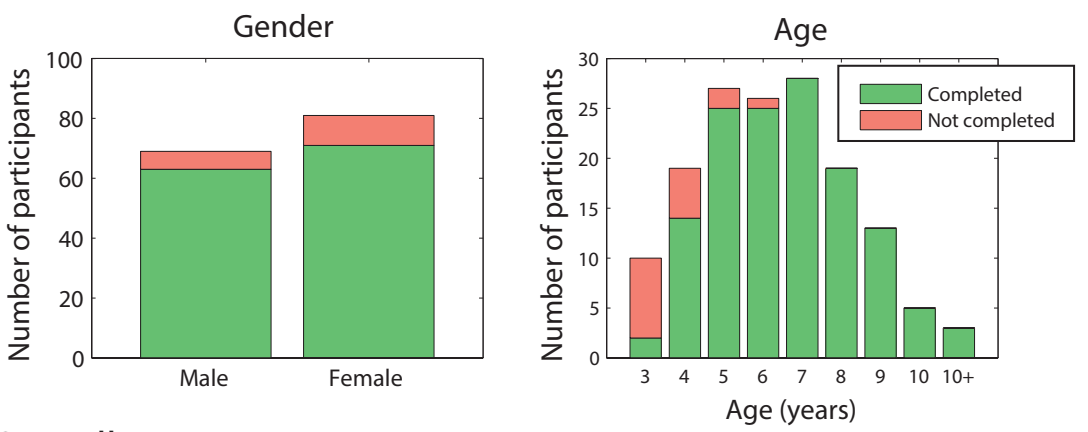

c) Overall scores
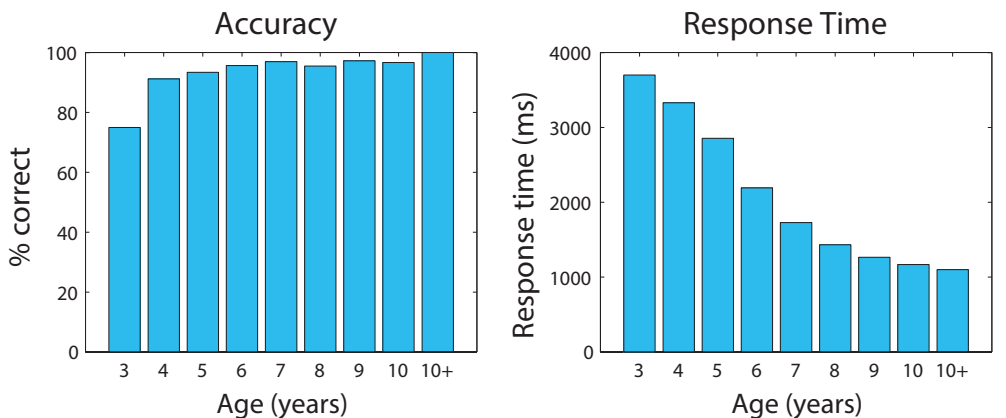

Figure 2. a) Illustration of the different response time measured considered. The initiation time is defined as the time needed to leave the start-box (red section). The movement time is the time between leaving the start-box and entering the response box (the box that was clicked; green section). The completion time or response time is the time between clicking on the start-box and clicking on the response box (all sections combined). b) Age and gender of the children, with in green the children who completed all trials of the study, and in red the children withdrawing before completing all trials. c) Overall accuracy and response times for the different age groups, showing that with increasing age accuracy increases and response times ('completion times') decrease. 
$\left.F(1,133)=6.95, p=0.028, \eta^{2}=0.050\right)$. Incongruent hands significantly distracted from responding to arrows, whereas no such effects were found of incongruent eyes (Figure 3a). Distraction effects of arrows and eyes did not differ significantly for responses to hands (interaction: $\left.F(1,133)=0.83, p>0.99, \eta^{2}=0.0062\right)$, and neither cue distracted significantly with moving out of the start box (Figure 3a).

To examine whether congruency effects depended on age, a three-way ANOVA examined the effects of age, target-distractor combination and congruency. The non-significant three-way interaction showed that age did not influence the congruency effects across targetdistractor combinations $\left(F(5,620)=1.16, p=0.26, \eta^{2}=0.052\right)$. Bonferroni corrected follow-up ANOVAs, testing the interaction between age and congruency for each of the target-cue combinations, showed no influence of age on distraction for any of the targetdistractor combinations (Figure $3 b$ ).

Completion times. Completion times are defined as the time from clicking the START button to entering the correct response box. As for initiation times, completion times were longer when responding to eyes than to hands $(t(133)=4.06, p<0.001, d=0.35)$ and arrows $(t(133)=2.49, p=0.042, d=0.26)$. Completion times for hands and arrows did not differ significantly $(t(133)=1.26, p=0.64, d=0.11)$.

The distraction effects of hands and arrows on eyes differed significantly $(F(1,129)$ $\left.=10.8, p=0.0039, \eta^{2}=0.077\right)$. Larger distraction effects were found from arrows than from hands (Figure 4a). Distraction effects were weaker for eyes as distractors, both when responding to arrows $\left(F(1,131)=18.8, p<0.001, \eta^{2}=0.13\right)$ and when responding to hands $\left(F(1,131)=12.7, p=0.0016, \eta^{2}=0.087\right)$. While hands and arrows exerted significant distraction on each other, eyes did not significantly distract either of these cues (Figure 4a).

Across all six target-cue combinations, no effect of age on distraction effects were found (absent three-way interaction: $F(5,610)=0.90, p=0.62)$. As shown by the p-values in Figure 3b, none of the individual interactions with age survived Bonferroni correction. 
a) Initiation times, across all participants
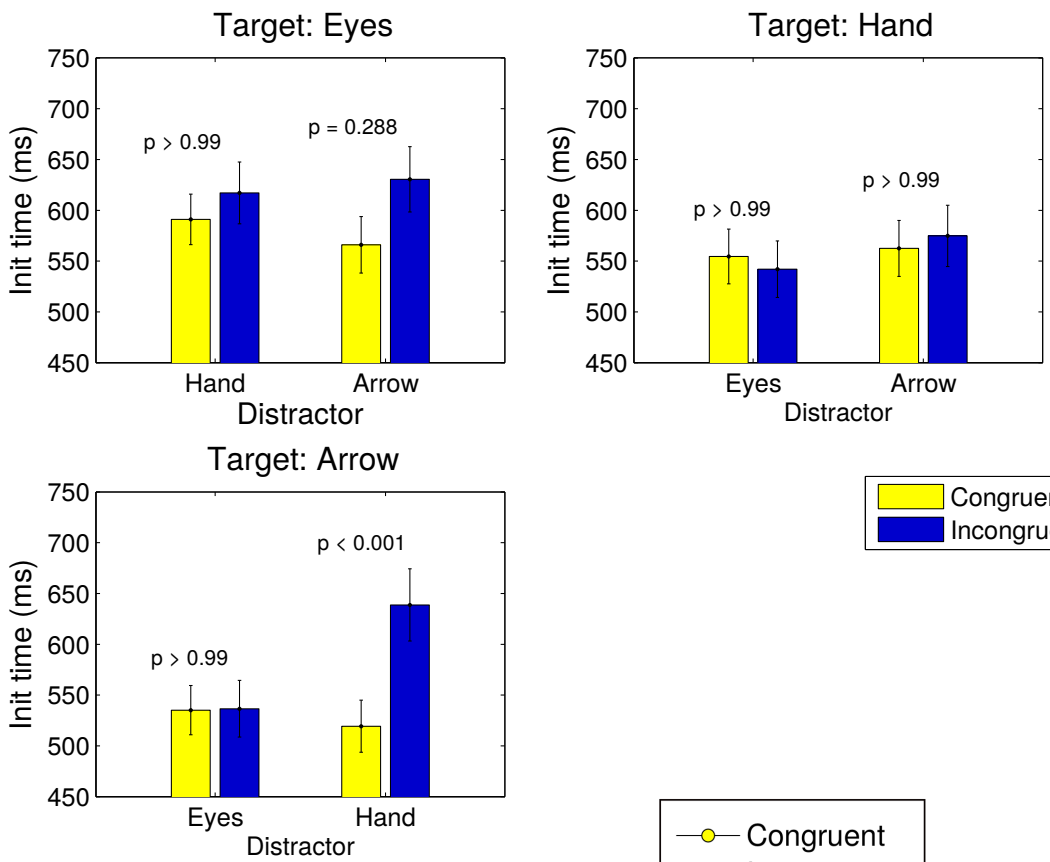

b) Initiation times, per age group
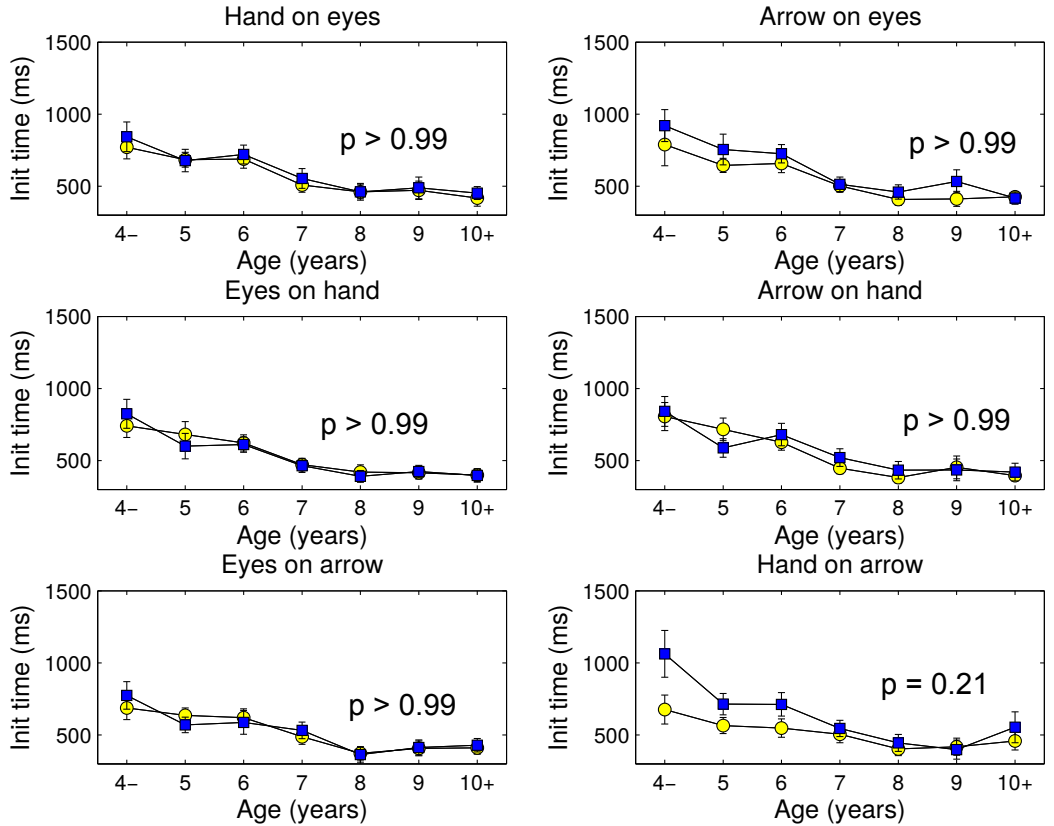

Figure 3. a) Time needed to move the mouse out of the START box ('initiation times') for each target and distractor combination, across all participants. b) Initiation times for each age group and target and distractor combination. Each graph shows the Bonferroni corrected p-value for the comparison between congruent and incongruent distractor conditions (a) or the interaction between age and congruency (b). ' $\mathrm{p}>0.99$ ' denotes situations where the Bonferroni corrected p-value was larger than 1. 


\section{a) Completion times, across all participants}
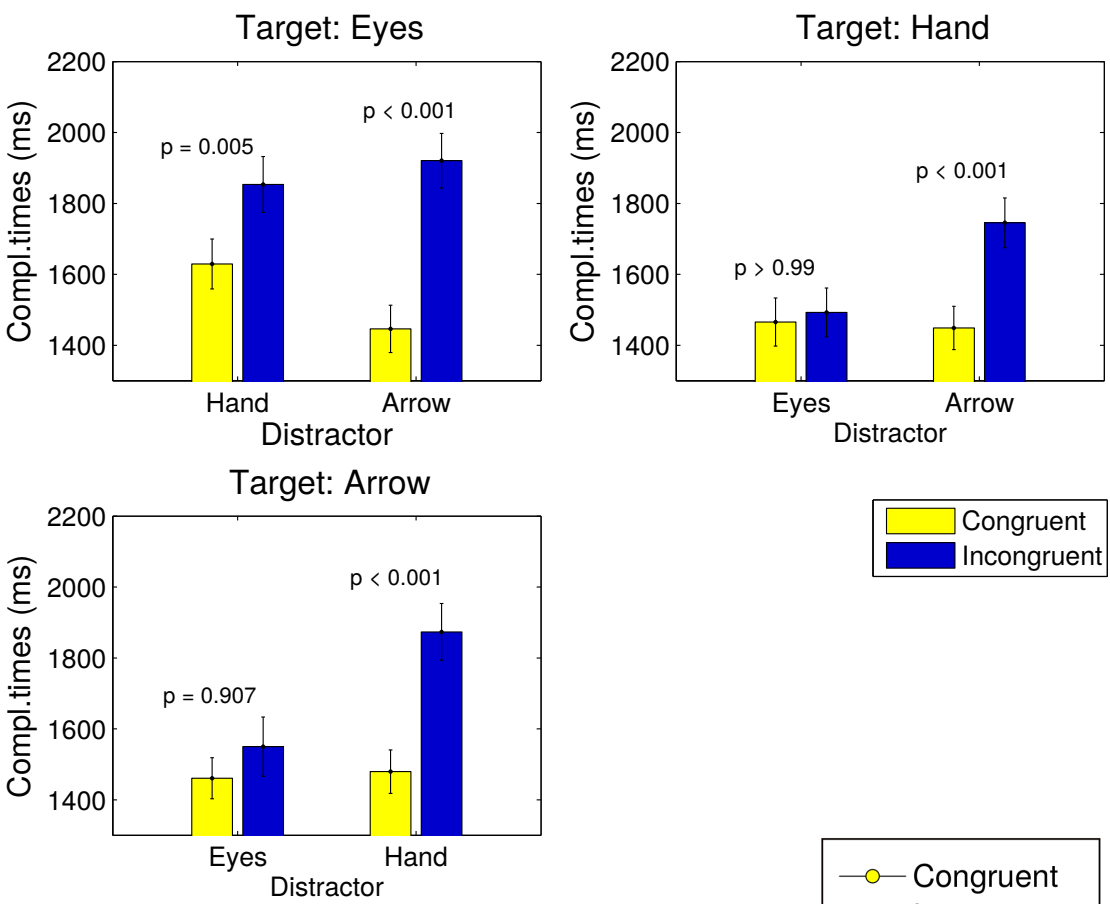

\section{b) Completion times, per age group}
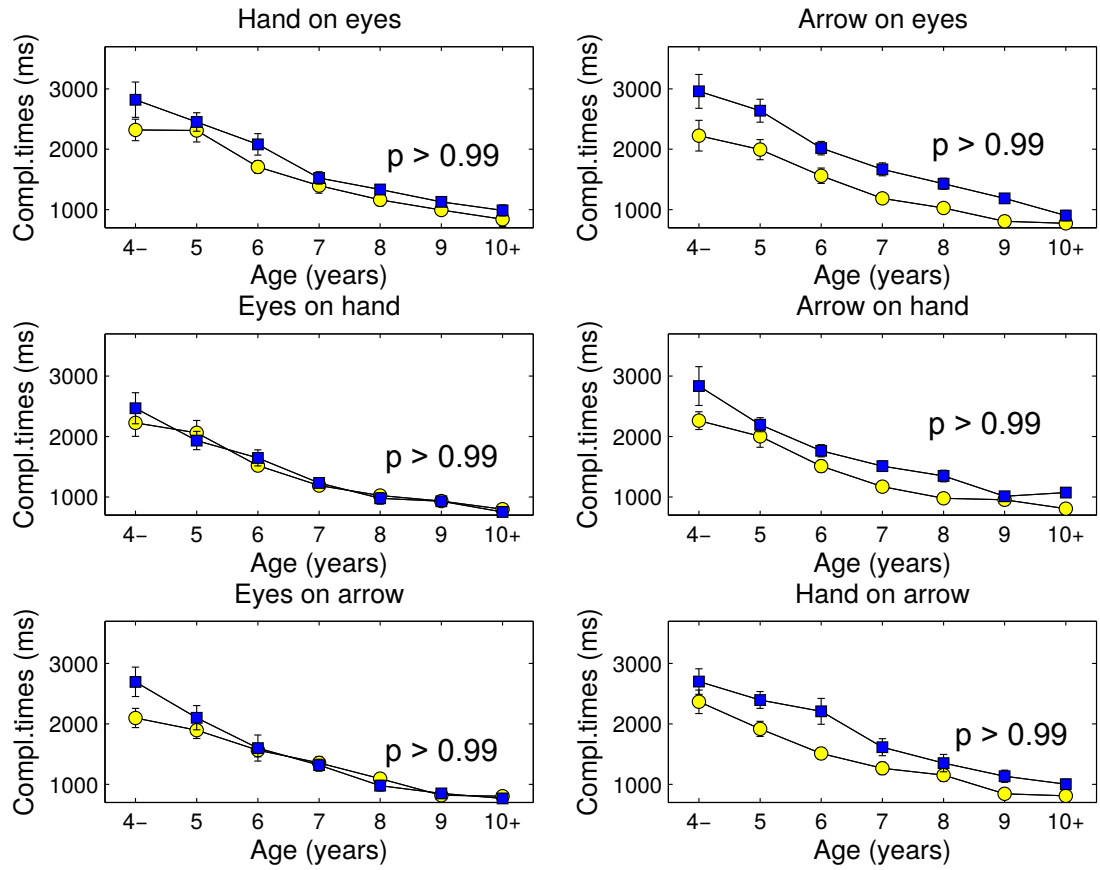

Figure 4. a) Time needed to enter the target box ('completion times'), for each target and distractor combination, across all participants. b) Time to enter the target button ('completion times') for the different age groups and target and distractor combinations. Each graph shows the Bonferroni corrected p-value for the comparison between congruent and incongruent distractor conditions (a) or the interaction between age and congruency (b). ' $\mathrm{p}>0.99$ ' denotes situations where the Bonferroni corrected p-value was larger than 1. 
Movement times. Figure 5 shows the movement times, which are defined as the time from leaving the START button to entering the selected response button. Movement times did not differ when responding to eyes and arrows $(t(133)=2.27, p=0.074, d=0.19)$ and to arrows and hands $(t(133)=1.10, p=0.083, d=0.095)$. Movement times were shorter when responding to hands to to eyes $(t(133)=3.13, p=0.0006, d=0.27)$.

Arrows had larger distraction effects on eyes than hands $(F(1,129)=10.5, p=0.0045$, $\eta^{2}=0.075$ ). Eyes were weaker distractors than hands (when responding to arrows, $F(1,131)$ $=11.0, p=0.0036, \eta^{2}=0.077$ ) and arrows (when responding to hands, $F(1,133)=8.64$, $\left.p=0.012, \eta^{2}=0.061\right)$. Whereas hands and arrows significantly distracted from responses on the other cues, no such distraction effects were found of eyes (Figure 5).

Across all target-cue combinations, congruency did not interact with age (three-way interaction: $\left.F(5,61)=0.97, p=0.52, \eta^{2}=0.042\right)$. As shown by the p-values in Figure $5 \mathrm{~b}$, none of the individual interactions between age and congruency reaches statistical significance after Bonferroni correction.

\section{Mouse trajectory measures}

Maximum deviation. Figure 6a illustrates how maximum deviation is determined. To make the measure independent of the dimensions of the screen and the locations of the stimuli, it is expressed as a percentage of the length of the straight path trajectory between the start and end of the mouse trajectory (see Nummenmaa \& Hietanen, 2006). Bonferroni corrected paired samples t-tests show that maximum deviations were not significantly different for arrow and hand targets $(t(133)=1.46, p=0.44, d=0.13)$ and for eyes and arrow targets $(t(133)=1.58, p=0.45, d=0.14)$. Peak deviations to eyes were significantly larger than those to hands $(t(133)=3.34, p=0.003, d=0.29)$, possibly due to stronger distraction in the incongruent distractor conditions.

Arrows led to larger maximum deviations than hands when responding to eyes $\left(F(1,129)=9.14, p<=0.01, \eta^{2}=0.066\right)$, but significantly larger deviations for incongruent distractors were found for both distractor types (Figure 6b). Eyes resulted in weaker 


\section{a) Movement time, across all participants}
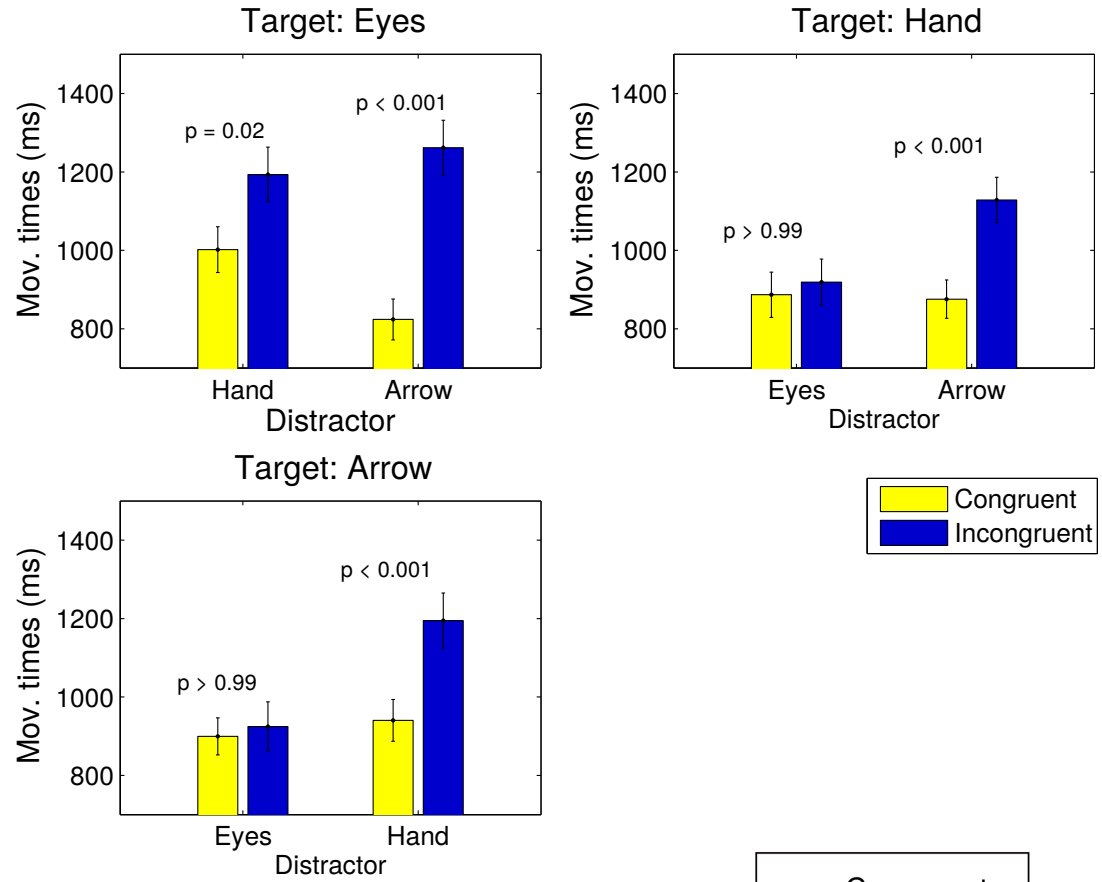

b) Movement time, per age group
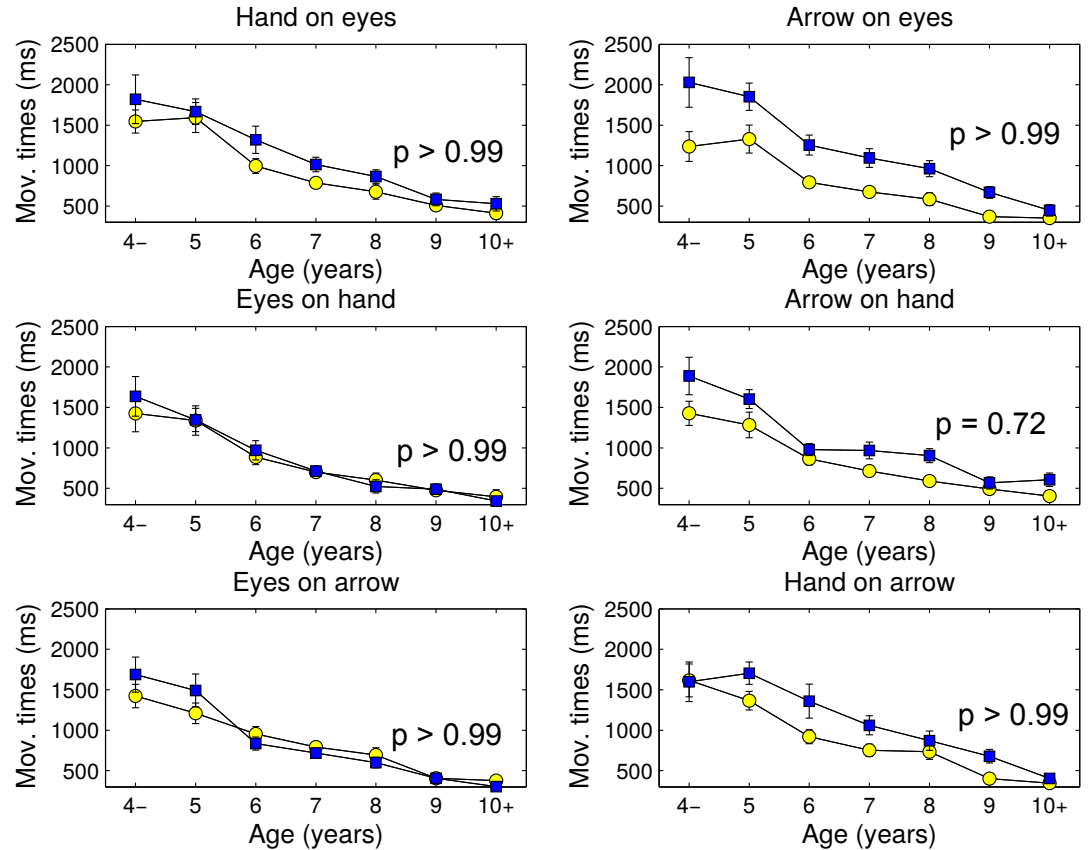

Figure 5. a) Time from leaving the 'start' box until reaching the target box ('movement times'), for each target and distractor combination, across all participants. b) Time from the 'start' box until the target box ('movement times') for the different age groups and target and distractor combinations. Each graph shows the Bonferroni corrected p-value for the comparison between congruent and incongruent distractor conditions (a) or the interaction between age and congruency (b). ' $p>0.99$ ' denotes situations where the Bonferroni corrected p-value was larger than 1. 
distraction than arrows (responding to hands, $F(1,133)=22.1, p<0.001, \eta^{2}=0.014$ ) and hands (responding to arrows, $F(1,31)=18.7, p<0.001, \eta^{2}=0.012$ ). Eyes did significantly distract responses to arrows (Figure 6b).

Across all target-distractor combinations, no effect of age on distraction was found (three-way interaction: $F(5,610)=1.16, p=0.26, \eta^{2}=0.048$, and for none of the targetdistractor combinations, the interaction between age and congruency reached statistical significance (Figure 6c).

Time to peak. Figure 7a illustrates how time to peak was computed. It measures the distance covered across the straight path between start and end until the maximum deviation occurs. Time to peak did not differ between responding to eyes and arrows $(t(133)=1.87, p>0.99, d=0.063)$, between responding to eyes and hands $(t(133)=1.33$, $p=0.56, d=0.11)$ and between responding to arrows and hands $(t(133)=0.54, p>0.99$, $d=0.047)$.

No significant difference was found in the distraction from hands and arrows when responding to eyes $\left(F(1,129)=0.045, p>0.99, \eta^{2}=0.00035\right)$. As Figure $7 \mathrm{~b}$ shows that for both distractors, time to peak was significantly larger for congruent than incongruent distractor cues. As in Hermens et al. (2017), time to peak shows an opposite pattern to maximum deviation (large deviations are associated with early peaks). Eyes and hands showed similar distraction on arrows $\left(F(1,131)=1.78, p=0.55, \eta^{2}=0.013\right)$. Distraction from arrows and eyes on hands did not differ significantly either $(F(1,133)=2.16, p=0.43$, $\eta^{2}=0.016$ ). Arrows significantly distracted responses to hands (Figure 7b).

Age did not have an influence on distraction effects on times to peak (three-way interaction: $\left.F(5,610)=0.92, p=0.59, \eta^{2}=0.042\right)$. Tested for each target-distractor combination separately, no interaction between age and congruency was found (Figure 7c).

Turn around trajectories. A second trajectory measure of distraction is the percentage of trajectories strongly deviating towards the alternative response box ('turn-around'). As a cut-off of for such strong deviations $40 \%$ of the straight path length was chosen on the 
a) Definition of maximum deviation

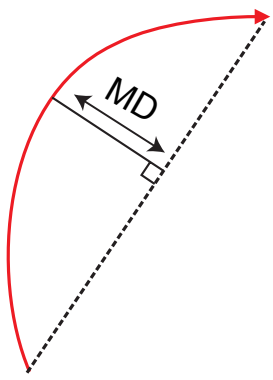

b) Maximum deviation, across all participants
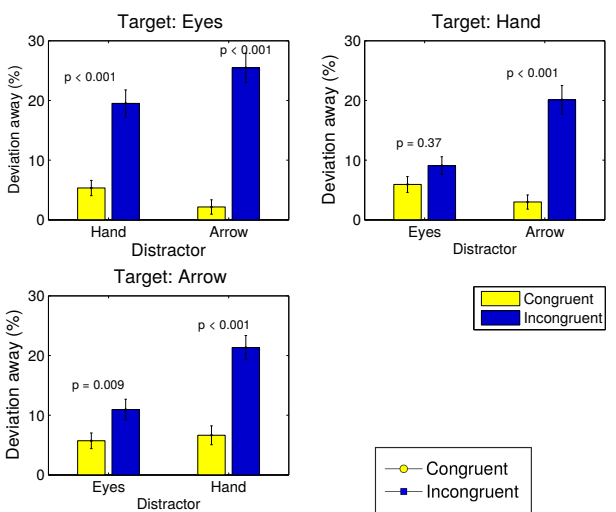

Maximum deviation, per age group
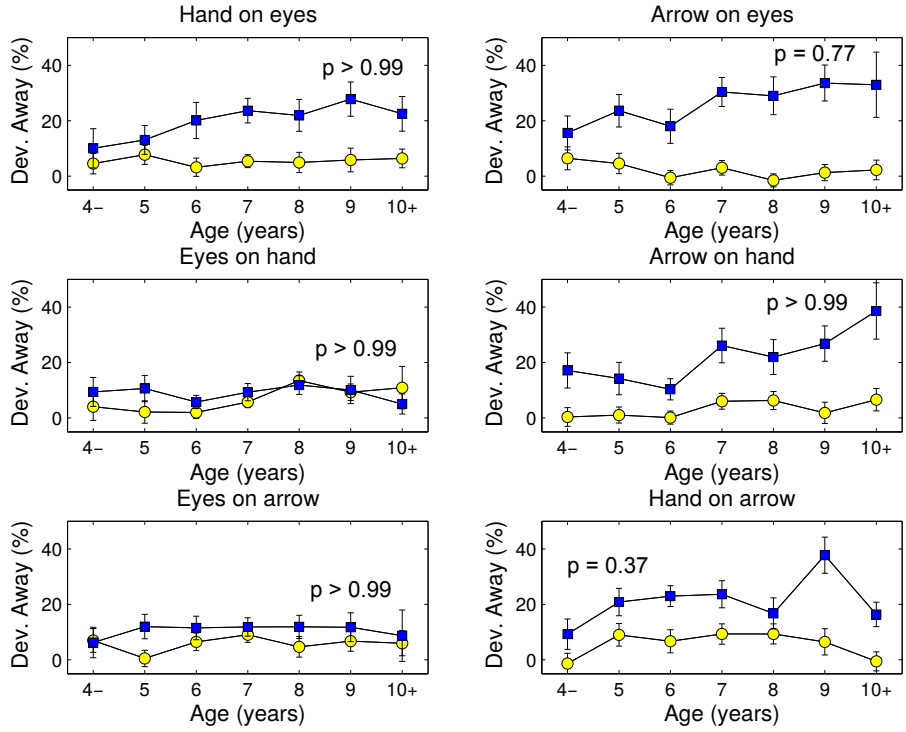

Figure 6. a) Definition of maximum deviation as the largest perpendicular distance to the straight path from start to end of the mouse movement, as a percentage of straight path length. b) Maximum deviation across all participants, for each target and distractor combinations. c) Maximum deviation as a function of age, across target and distractor combinations. Each graph shows the Bonferroni corrected p-value for the comparison between congruent and incongruent distractor conditions (a) or the interaction between age and congruency (b). ' $p>0.99$ ' denotes situations where the Bonferroni corrected p-value was larger than 1. 
b) Time to peak, across all participants
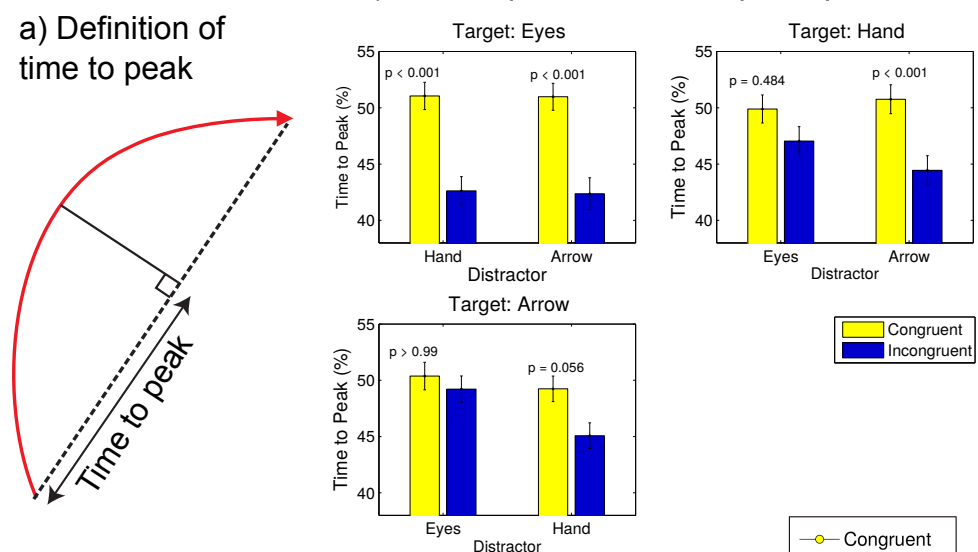

c) Time peak, per age group
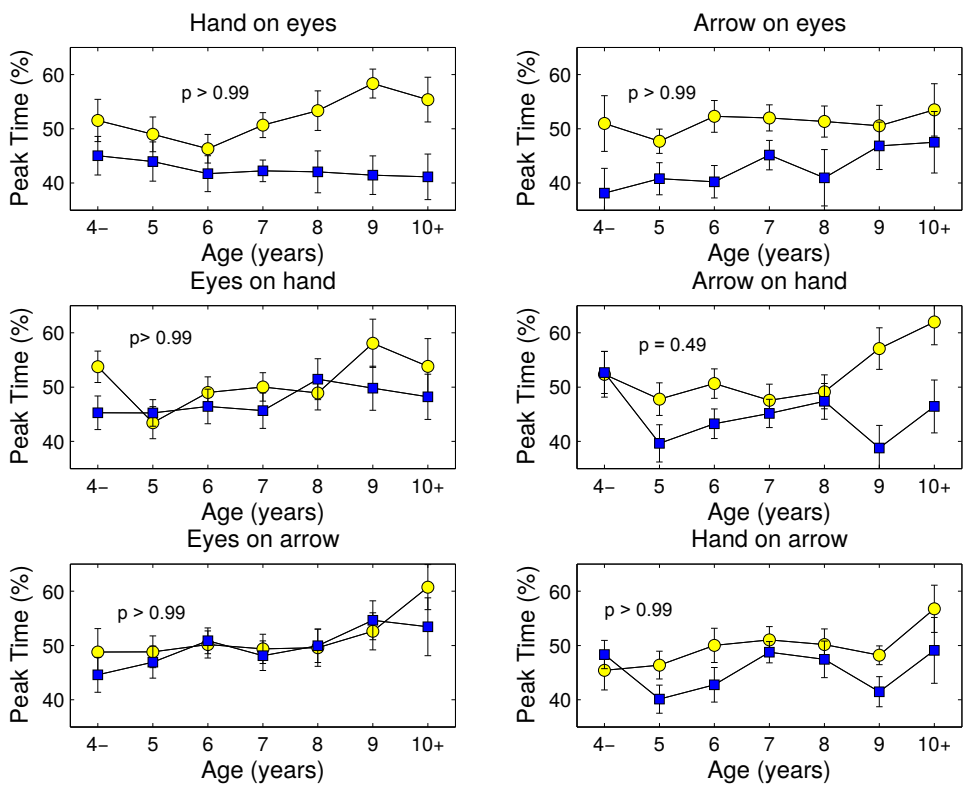

Figure 7. a) Definition of time to peak, as a percentage of the straight path where the maximum deviation occurs. b) time to peak for all participants, for each target and distractor combination. c) time to peak as a function of age for each target-distractor combination. Each graph shows the Bonferroni corrected p-value for the comparison between congruent and incongruent distractor conditions (a) or the interaction between age and congruency (b). ' $p>0.99$ ' denotes situations where the Bonferroni corrected p-value was larger than 1. 
basis of a histogram of maximum deviations, but similar patterns of results were obtained when other cut-off values (e.g., $50 \%$, or $60 \%$ ) were used. Turn-around percentages were no different when responding to eyes and arrows $(t(133)=2.15, p=0.099, d=0.19)$ and when responding to arrows and hands $(t(133)=1.18, p=0.72, d=0.10)$. Larger percentages of turn-around trajectories were found when responding to eyes than to hands $(t(133)=3.81$, $p=0.001, d=0.33)$.

Distraction effects from hands and arrows on eyes did not differ significantly $(F(1,129)$ $\left.=4.90, p=0.086, \eta^{2}=0.037\right)$. As shown in Figure 8b, both types of distractors significantly interfered with eyes as targets. Distraction effects from eyes on arrows were significantly weaker than those from hands $\left(F(1,131)=14.4, p<0.001, \eta^{2}=0.099\right)$, and distraction from eyes on hands did not reach statistical significance (Figure 8b). Distraction effects from eyes were also significantly weaker than those of arrows and hand targets $(F(1,133)$ $\left.=30.4, p<0.001, \eta^{2}=0.19\right)$, but here significant distraction effects of eyes were found (Figure 8c).

Across all target-distractor combinations, congruency did not interact with age (threeway interaction: $\left.F(5,610)=0.92, p=0.59, \eta^{2}=0.038\right)$. As shown in Figure $8 \mathrm{c}$, for none of the target-distractor combinations, age interacted significantly with congruency.

\section{Distraction compared}

The analysis so far has used a large number of age groups (4-, 5, 6, 7, 8, 9, 10+), which may have lowered the statistical power to detect any group differences. In a final analysis, larger age groups were used (3-5 years, 6-7 years, 8-11 years). Using larger age groups may reduce sensitivity to age effects that occur for a very specific age range (e.g., particularly strong cueing by eyes at 3 years of age), but the data plots so far have not suggested such specific age effects. To reduce the number of factors in the analysis and in the data plots (see Figure 9), congruency effects were reduced to the difference between the incongruent and incongruent condition (to match the direction of these differences, the difference between congruent and incongruent conditions was taken for the time to 
b) Turn-around trajectories, across all participants

a) Definition of turn-around trajectories

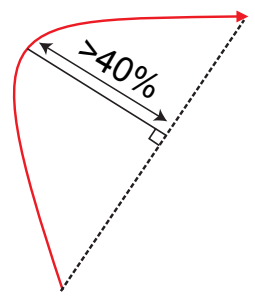

Target: Eyes

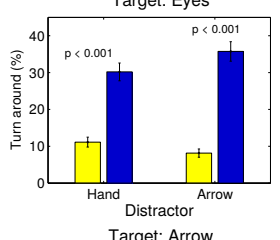

Target: Arrow

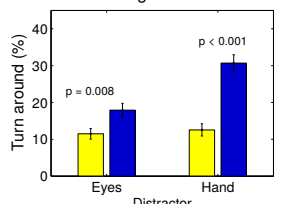

Target: Hand
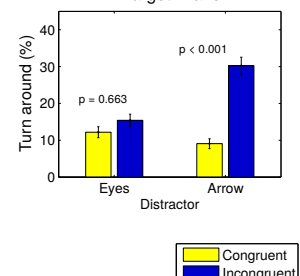

\section{- Congruent}

- Incongruent

c) Turn-around trajectories, per age group
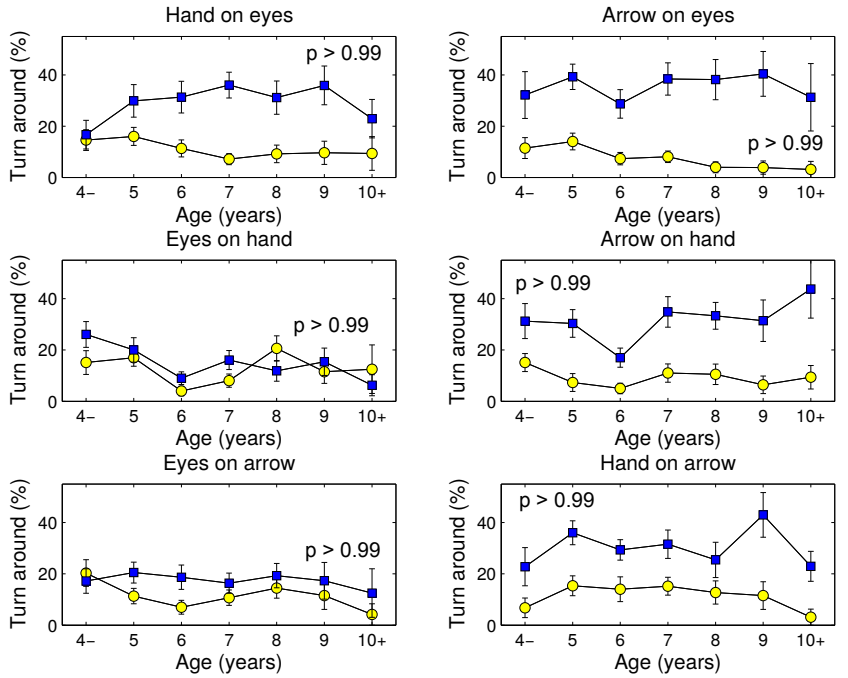

Figure 8 . a) Turn around trajectories were defined as trajectories where the peak deviation was larger than $40 \%$ of the straight path length. b) Turn around frequencies for all participants, for each target and distractor combination. c) Turn around frequencies per age, target and distractor combination. Each graph shows the Bonferroni corrected p-value for the comparison between congruent and incongruent distractor conditions (a) or the interaction between age and congruency (b). 'p>0.99' denotes situations where the Bonferroni corrected p-value was larger than 1. 
peak measure). Figure 9 shows that for most target-distractor combinations, measures and age groups, distraction effects were positive (more distraction in the incongruent than in the congruent condition). Distraction effects were weakest and least consistent for initiation times (the time to move the mouse out of the START box), which suggests that participants started their mouse movement quickly and then started making their decision. For many of the response time measures, distraction effects appear to be strongest for the younger age groups. For the trajectory measures, the opposite pattern appears to occur, which may suggest that younger children slowed down their movements in situations of conflict, whereas older children allowed their mouse trajectories to deviate to avoid slowing down.

For initiation times, there was a significant interaction between target-distractor combination and age group $\left(F(10,630)=1.87, p=0.046, \eta^{2}=0.028\right)$. Posthoc ANOVAs testing the effect of age for each of the target-distractor conditions suggest that this interaction was due to a near significant effect (after Bonferroni correction) of age for the hand-arrow combination (p-values in Figure 9). For completion times, there was no interaction between target-distractor combination and age group $\left(F(10,630)=1.03, p=0.42, \eta^{2}=0.015\right)$, but there were significant main effects of target-distractor combination $(F(5,630)=9.27, p<$ $\left.0.001, \eta^{2}=0.067\right)$ and of age $\left(F(2,126)=3.59, p=0.031, \eta^{2}=0.054\right)$. However, for none of the target-distractor combinations the effect of age survived Bonferroni correction (Figure 9). For movement times, there was no interaction between target-distractor combination and age $\left(F(10,630)=1.24, p=0.26, \eta^{2}=0.018\right)$, but there were significant main effects of target-distractor combination $\left(F(5,630)=9.02, p<0.01, \eta^{2}=0.066\right)$ and age $\left(F(2,126)=3.45, p=0.035, \eta^{2}=0.052\right)$. Tested for each target-distractor combination separately, significantly more distraction was found from eyes on arrows for the youngest age group (Figure 9).

For maximum deviation, the interaction between target-distractor combination and age approached statistical significance $\left(F(10,630)=1.82, p=0.054, \eta^{2}=0.025\right)$, and there was a significant main effect of target-distractor combination $\left(F(5,630)=15.0, p<0.01, \eta^{2}\right.$ $=0.10)$. No main effect of age was found $\left(F(2,126)=2.20, p=0.12, \eta^{2}=0.034\right)$, and no 
Table 1: Overview of target comparison results (e.g., 'are children responding more quickly to eyes than to hands?'). Cells with cue names indicate the target that had lower response times, smaller peak deviations, later times to peak and fewer turn-around trajectories (the stronger cues). Nonsignificant differences are shown as 'NS'.

\begin{tabular}{llll}
\hline $\begin{array}{l}\text { Dependent } \\
\text { measures }\end{array}$ & $\begin{array}{l}\text { Eyes } \\
\text { versus } \\
\text { Hands }\end{array}$ & $\begin{array}{l}\text { Eyes } \\
\text { versus } \\
\text { Arrows }\end{array}$ & $\begin{array}{l}\text { Hands } \\
\text { versus } \\
\text { Arrows }\end{array}$ \\
\hline Initiation time & Hands & Arrows & NS \\
$\begin{array}{l}\text { Completion time } \\
\text { Movement time }\end{array}$ & Hands & Arrows & NS \\
$\begin{array}{l}\text { Peak deviation } \\
\text { Time to peak }\end{array}$ & Nands & NS & NS \\
$\begin{array}{l}\text { Turn around } \\
\text { trajectories }\end{array}$ & Hands & NS & NS \\
\hline
\end{tabular}

significant effect of age was found for each of the target-distractor combinations ( $p$-values in Figure 9). For time to peak, the interaction between target-distractor combination and age was not statistically significant $\left(F(10,630)=0.60, p=0.82, \eta^{2}=0.0091\right)$, but there was a significant main effect of target-distractor combination $(F(5,630)=3.54, p<0.01$, $\left.\eta^{2}=0.027\right)$. There was no main effect of age $\left(F(2,126)=0.82, p=0.44, \eta^{2}=0.013\right)$, and no significant effects of age were found for each of the target-distractor combinations (Figure 9). Finally, for the percentage of turn-around trajectories, the interaction between target-distractor combination and age was not statistically significant $(F(10,630)=1.56, p$ $\left.=0.12, \eta^{2}=0.021\right)$. There was a significant main effect of target-distractor combination $\left(F(5,630)=16.0, p<0.01, \eta^{2}=0.11\right)$, but no main effect of age was found $(F(2,126)$ $\left.=0.63, p=0.53, \eta^{2}=0.0099\right)$. No significant effects of age were found for each of the target-distractor combinations either (Figure 9). Together these results suggest that some small age-effects can be detected if larger groups are used (particularly for the response time measures), but these effects are not consistent across measures and are generally associated with small effect sizes.

\section{Discussion}

Studies of social attention have typically presented stimuli where participants are already looking (e.g., Driver et al., 1999; Kuhn \& Kingstone, 2009; Frischen et al., 2007). Such studies suggest that attention shifts from perceived eye gaze cues are at least of the 
a) Response time effects
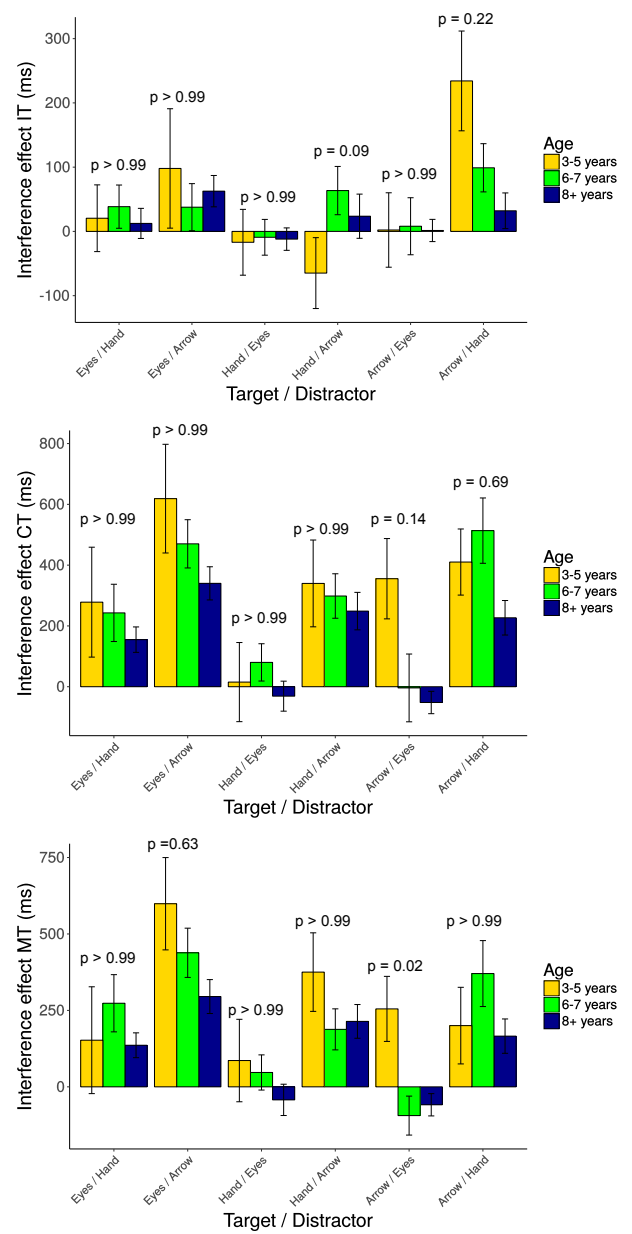

b) Trajectory effects
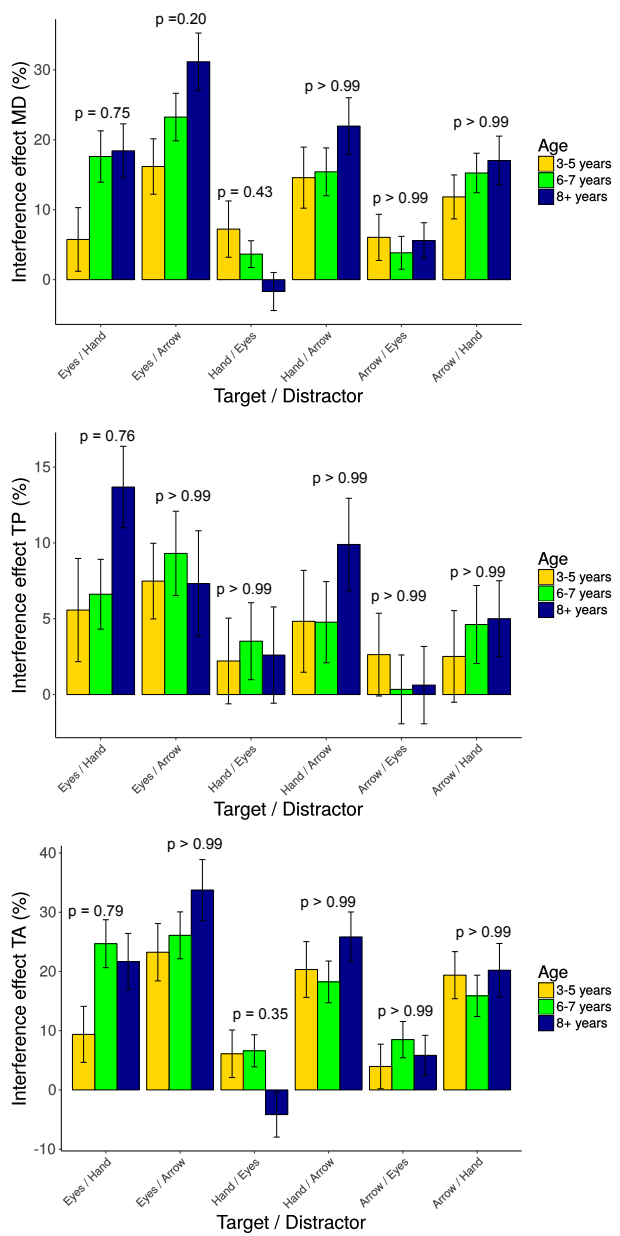

Figure 9. Distraction effects (incongruent condition minus the congruent condition, except for time to peak (TP), where the reverse is shown) across age groups. a) Distraction effects for response times measures (IT $=$ initiation times, $\mathrm{CT}=$ completion times, $\mathrm{MV}=$ movement times $)$. b) Distraction effects for trajectory measures ( $\mathrm{MD}=$ maximum deviation, $\mathrm{TP}=$ time to peak, $\mathrm{TA}=$ turn-around). Error bars show the standard error of the mean across participants. P-values in the graphs indicate the Bonferroni corrected (six comparisons) effects of age. 
Table 2: Overview of Distraction effects, indicating for each target-distractor combination, whether the distractor significantly interfered with responses to the target across all children (first letter - X for yes, $\mathrm{O}$, for no) and whether this distraction interacted with age (second letter - X for yes, O, for no), based .

\begin{tabular}{lllllll}
\hline $\begin{array}{l}\text { Dependent } \\
\text { measures }\end{array}$ & $\begin{array}{l}\text { Hands on } \\
\text { Eyes }\end{array}$ & $\begin{array}{l}\text { Arrows } \\
\text { on Eyes }\end{array}$ & $\begin{array}{l}\text { Eyes on } \\
\text { Hands }\end{array}$ & $\begin{array}{l}\text { Arrows } \\
\text { on Hands }\end{array}$ & $\begin{array}{l}\text { Eyes on } \\
\text { Arrows }\end{array}$ & $\begin{array}{l}\text { Hands on } \\
\text { Arrows }\end{array}$ \\
\hline Initiation time & OO & OO & OO & OO & OO & XO \\
$\begin{array}{l}\text { Completion time } \\
\text { Movement time }\end{array}$ & OO & XO & OO & XO & OO & XO \\
$\begin{array}{l}\text { Peak deviation } \\
\text { Time to peak }\end{array}$ & XO & XO & OO & XO & OO & XO \\
$\begin{array}{l}\text { Turn around } \\
\text { trajectories }\end{array}$ & XO & XO & OO & XO & XO & XO \\
\hline
\end{tabular}

same magnitude, or stronger than attention shifts from arrow cues. We have recently shown that when stimuli are presented away from fixation, but allowing participants to make an eye movement to the stimulus, the influence of eye gaze cues is strongly reduced, while influences from arrows and pointing hands remain strong (Hermens et al., 2017). This finding is important, because in day-to-day vision cues are not always directly fixated. The present study shows that these stronger influences of arrows and pointing hands, compared to eye gaze cues, are also found in children (who can be expected to be less strongly exposed to arrow cues, compared to adults), and that these effects are independent of participants' age. These results suggest that, also in children, the shape of the cue is more important the the biological relevance in situations where the cue is not presented at fixation.

A mouse tracking paradigm was employed (Freeman \& Ambady, 2010; Freeman, Dale, \& Farmer, 2011; Hehman, Stolier, \& Freeman, 2015), as our previous study (Hermens et al., 2017) had demonstrated that distraction from simultaneously presented incongruent cues (in adults) can only be reliably detected using mouse trajectories. Mouse tracking yields a wealth of information. Here, the focus was on six measures extracted from the mouse trajectories: Three measures related to response times, and three measures related to the shape of the mouse trajectories. The results for the various measures are summarized in Tables 1 and 2. Table 1 compares the cues as targets, indicating which cue was strongest in terms of shorter response times, longer times to peak, larger peak deviations, and the number of reversed trajectories ('turn-around'). This table suggests that arrows and hands 
are stronger than eye gaze cues, and that response times are a more sensitive measure of cue strength when acting as the response target (in agreement with Hermens et al., 2017). Table 2 indicates whether the distractor cue significantly interfered with the target cue (first letter in the letter combination; $\mathrm{X}$ in the case of significant distraction, $\mathrm{O}$ when distraction was not found) and whether this distraction was dependent on the age group of the participants (the second letter in the letter combination; $\mathrm{X}$ for age dependent distraction and $\mathrm{O}$ of age independent distraction). This table suggests that trajectory measures are better at detecting distraction from simultaneously presented incongruent cues than response time measures (in agreement with data in adults Hermens et al., 2017) and that such distraction effects are largely independent of age. Finally, arrows and hands interfered more often with target cues (in particular with eyes) than eyes interfered with arrows or hands. The various mouse tracking measures, in line with the previous adult data (Hermens et al., 2017), therefore provide consistent results.

The strong cueing by arrows in children in the present study is in line with past work on the effects of cues presented at fixation. Using line arrows, Ristic et al. (2002) found similar cueing in 3 to 5 year olds by arrows as by a line drawing of a gazing face. Likewise, for unpredictive gray arrow cues, Senju et al. (2004) found similar cueing as for photographs of faces in 11 years olds. Kuhn et al. (2010) found similar cueing by line drawings of arrows and faces in 10 year olds. Jakobsen et al. (2013) found cueing by line drawings of arrows in 3 to 19 year olds, although for the youngest participants, cueing may have been based on the extra perceptual weight of the arrow head in the image (but if perceptual weight would play a role in the present study, the pointing hand should have yielded opposite cueing effects, while gazing eyes should have produced strong cueing effects). The one other study using an interference paradigm (Barnes et al., 2007) found interference from arrows and eyes in 6 to 13 years old, where stronger interference from eyes was only found in emotional faces and in older children (10 to 13 years).

The results raise the question why the strong interference from arrows occurs in younger age groups. Until studies are conducted in which the visual input of children 
across a broad age range is recorded and documents (e.g., by having children wear a headmounted camera, which also detects when the child has their eyes closed), it is uncertain whether children are indeed exposed less to arrows and more to biological cues (eye gaze, and pointing gestures) than adults. Exposure to arrows is more likely when navigating the surroundings, as arrows tend to occur in signs. While very young children are less likely to travel on their own, they may still be exposed to arrows when traveling with their parents. Possibly, children will also be seeing arrows in television shows and cartoons. And it could be the case that a little exposure suffices for cueing effects to occur, and that extensive exposure is not needed. Alternatively, the cueing effects are not driven by biological relevance, and by cue shape instead.

No clear advantage of pointing hands over arrows was found. This result appears to be at odds with previous findings suggesting that pointing hands influence children's eye movements more strongly than arrows (Gregory, Hermens, Facey, \& Hodgson, 2016). This advantage of hands over arrows, however, was particularly strong in the youngest age group (3 to 5 years), which was the age group where the present study often failed in having children complete the study or to do the task (using the mouse). Moreover, the arrow in the study by Gregory et al. (2016) was embedded in a round blue sign, and recent (Hermens, in press) and past studies (Nummenmaa \& Hietanen, 2009) have suggested that arrow signs embedded in a larger sign may not be as effective, possibly because their direction is more difficult to perceive than arrows shown immediately against the background. Finally, the work by Gregory et al. (2016) presented cues at fixation, which may have led to different effects of the various cues, compared to when cues are presented away from fixation (Hermens et al., 2017).

The present study introduced a few changes to the paradigm by Hermens et al. (2017). In the present study, a line drawing of a pointing hand was used, whereas in the past study in adults (Hermens et al., 2017), only photographs of pointing hands were used. The past study did use line drawings of (round) gazing eyes, as well as photographs of eyes. Whereas in the past study the arrow was filled (with a solid gray fill), the present study only used an 
outline for the arrow (to better the visual properties of the other cues). A further change involved the response boxes. Whereas the past study used the words 'LEFT' and 'RIGHT' inside the response boxes, the present study showed the target shape instead, aimed to make it easier for the children to understand the task. The use of symbols rather than words could have influenced the task that children were performing (a visual matching task, rather than a direction discrimination task). While the use of symbols could have an effect on responses to the targets, it cannot cannot explain distraction effects of the other cue on the screen (children must have coded their directions for such distraction effects to occur). Moreover, the highly similar results between the data from the adults (Hermens et al., 2017) and the present results suggest that using symbolics rather than words did not critically influence the results (or any of the other changes made).

The present study shows that mouse tracking is feasible in children. Past research found that mouse tracking can work in children of 4 years and 8 months of age, and 5 years and 10 months of age (Anderson, Farmer, Goldstein, Schwade, \& Spivey, 2011), but did not examine the age range across which children could perform a mouse tracking task. The present results suggest that mouse tracking can be used from the age of 4 , and possibly in older 3-year-olds. Children who were struggling with the task were able to point with their finger in the direction of the target, and younger children often asked whether they could use the screen as a touch screen. This suggests that touch screen mouse tracking or finger motion tracking may be feasible in even younger children. When using touch screen responses, it should be established whether dragging movements show the same kind of response conflict effects as have been found for mouse trajectories, as studies in adults have suggested differences in trajectories of swiping and dragging movements on a touchpad (Akamatsu \& MacKenzie, 2002). When using motion tracking to record finger trajectories, it will need to be investigated whether table top trajectories are needed, or whether interference effects can be equally well measured using 3D motion trajectories. 
Conclusion

The present study shows, in line with past findings in adults (Hermens et al., 2017) that, also in children, cues with a clear shape (arrows, pointing hands) have stronger influences on directional responses when these cues are not presented at fixation, and that these stronger influences are independent of age. It also shows that mouse tracking is feasible in younger children, although future studies may want to look into motion tracking or the use of touch screens as an alternative to be able to test even younger children.

\section{References}

Akamatsu, M., \& MacKenzie, I. S. (2002). Changes in applied force to a touchpad during pointing tasks. International Journal of Industrial Ergonomics, 29(3), 171-182.

Anderson, S. E., Farmer, T. A., Goldstein, M., Schwade, J., \& Spivey, M. (2011). Individual differences in measures of linguistic experience account for variability in the sentence processing skill of five-year-olds. Experience, variation, and generalization: Learning a first language (Trends in Language Acquisition Research, 7, 203-221.

Barnes, K. A., Kaplan, L. A., \& Vaidya, C. J. (2007). Developmental differences in cognitive control of socio-affective processing. Developmental Neuropsychology, 32(3), 787-807.

Baron-Cohen, S. (1995). The eye direction detector (edd) and the shared attention mechanism (sam): Two cases for evolutionary psychology. In Society for research in child development conference, new orleans, mar 1993. : Lawrence Erlbaum Associates, Inc.

Burton, A. M., Bindemann, M., Langton, S. R., Schweinberger, S. R., \& Jenkins, R. (2009). Gaze perception requires focused attention: evidence from an interference task. Journal of Experimental Psychology: Human Perception and Performance, 35(1), 108-118.

Butterworth, G., \& Jarrett, N. (1991). What minds have in common is space: Spatial mechanisms serving joint visual attention in infancy. British Journal of Developmental Psychology, 9(1), $55-72$.

Dale, R., Roche, J., Snyder, K., \& McCall, R. (2008). Exploring action dynamics as an index of paired-associate learning. Plos ONE, 3(3), e1728.

D'Entremont, B., Hains, S., \& Muir, D. (1997). A demonstration of gaze following in 3-to 6-montholds. Infant Behavior and Development, 20(4), 569-572.

Driver, J., Davis, G., Ricciardelli, P., Kidd, P., Maxwell, E., \& Baron-Cohen, S. (1999). Gaze perception triggers reflexive visuospatial orienting. Visual Cognition, 6(5), 509-540. 
Freeman, J. B., \& Ambady, N. (2010). Mousetracker: Software for studying real-time mental processing using a computer mouse-tracking method. Behavior Research Methods, 42(1), $226-241$.

Freeman, J. B., Dale, R., \& Farmer, T. A. (2011). Hand in motion reveals mind in motion. Frontiers in Psychology, 2.

Frischen, A., Bayliss, A. P., \& Tipper, S. P. (2007). Gaze cueing of attention: visual attention, social cognition, and individual differences. Psychological Bulletin, 133(4), 694.

Gregory, N. J., Hermens, F., Facey, R., \& Hodgson, T. L. (2016). The developmental trajectory of attentional orienting to socio-biological cues. Experimental Brain Research, 234(6), 13511362.

Hehman, E., Stolier, R. M., \& Freeman, J. B. (2015). Advanced mouse-tracking analytic techniques for enhancing psychological science. Group Processes $\& 3$ Intergroup Relations, 18(3), 384-401.

Hermens, F. (in press). The effects of social and symbolic cues on visual search: Cue shape trumps biological relevance. Psihologija.

Hermens, F., Bindemann, M., \& Burton, A. M. (2017). Responding to social and symbolic extrafoveal cues: Cue shape trumps biological relevance. Psychological Research, 81(1), 24-42.

Hoehl, S., Reid, V. M., Parise, E., Handl, A., Palumbo, L., \& Striano, T. (2009). Looking at eye gaze processing and its neural correlates in infancyimplications for social development and autism spectrum disorder. Child Development, 80(4), 968-985.

Hood, B. M., Willen, J. D., \& Driver, J. (1998). Adult's eyes trigger shifts of visual attention in human infants. Psychological Science, 9(2), 131-134.

Jakobsen, K. V., Frick, J. E., \& Simpson, E. A. (2013). Look here! the development of attentional orienting to symbolic cues. Journal of Cognition and Development, 14(2), 229-249.

Kuhn, G., Benson, V., Fletcher-Watson, S., Kovshoff, H., McCormick, C. A., Kirkby, J., et al. (2010). Eye movements affirm: automatic overt gaze and arrow cueing for typical adults and adults with autism spectrum disorder. Experimental Brain Research, 201(2), 155-165.

Kuhn, G., \& Kingstone, A. (2009). Look away! eyes and arrows engage oculomotor responses automatically. Attention, Perception, \& Psychophysics, 71(2), 314-327.

Langton, S. R. (2000). The mutual influence of gaze and head orientation in the analysis of social attention direction. The Quarterly Journal of Experimental Psychology: Section A, 53(3), $825-845$.

Ludwig, C. J., \& Gilchrist, I. D. (2002). Measuring saccade curvature: A curve-fitting approach. Behavior Research Methods, 34(4), 618-624.

Mathôt, S., Schreij, D., \& Theeuwes, J. (2012). Opensesame: An open-source, graphical experiment 
builder for the social sciences. Behavior Research Methods, 44 (2), 314-324.

Nummenmaa, L., \& Hietanen, J. K. (2006). Gaze distractors influence saccadic curvature: Evidence for the role of the oculomotor system in gaze-cued orienting. Vision research, 46(21), 36743680.

Nummenmaa, L., \& Hietanen, J. K. (2009). How attentional systems process conflicting cues. the superiority of social over symbolic orienting revisited. Journal of Experimental Psychology: Human Perception and Performance, 35(6), 1738-1754.

Ristic, J., Friesen, C. K., \& Kingstone, A. (2002). Are eyes special? it depends on how you look at it. Psychonomic Bulletin 83 Review, 9(3), 507-513.

Senju, A., Tojo, Y., Dairoku, H., \& Hasegawa, T. (2004). Reflexive orienting in response to eye gaze and an arrow in children with and without autism. Journal of Child Psychology and Psychiatry, 45(3), 445-458.

Tipples, J. (2008). Orienting to counterpredictive gaze and arrow cues. Perception \& Psychophysics, $70(1), 77-87$. 